\title{
MATHEMATICAL MODELLING OF PROCESS OF FLUORATION OF METAL TUNGSTEN
}

\author{
Roman V. Brendakov ${ }^{1, ~}{ }^{*}$, Alexander V. Shvab ${ }^{1}$, and Vladimir N. Brendakov ${ }^{2}$ \\ ${ }^{1}$ National Research Tomsk State University, 634030 Tomsk, Russia \\ ${ }^{2}$ Seversk Technological Institute NRNU MEPhI, 636070 Seversk, Russia
}

\begin{abstract}
Wide use of products from metal tungsten in various branches of a national economy is caused by unique properties of this metal. By means of physical and mathematical modelling of process of receiving gaseous hexafluoride of tungsten it is possible to solve a problem of creation of import-substituting technology of processing of metal tungsten. The fluoride technology of processing of tungsten allows to put various coverings and to make products of tungsten of a various form which can't be received by traditional methods. Fluoride technology of processing of tungsten it is possible to present in the form sequences of two processes, process of fluorination of metal tungsten and process of restoration of fluorides of tungsten hydrogen.
\end{abstract}

\section{Introduction}

The process of fluorination of metal tungsten is carried out according to the chemical reaction given as

$$
W+3 F_{2}=W F_{6}
$$

To realize fluorination process, gaseous fluorine is passed over a layer of metal tungsten. Passing through the active zone of the reactor, fluorine interacts with tungsten powder to form tungsten hexafluoride $\left(W F_{6}\right)$. Consequently, the concentration of fluorine in the gas stream gradually decreases due to the decreasing amount of fluorine and its dilution by tungsten hexafluoride formed. The reduction of concentration of fluorine in the twocomponent mix would slow down the reaction of fluorination. However, the stoichiometric coefficient in formula (1) suggests a reduction of the speed of the gas flow. In view of the limiting factors, which exert significant effect on the speed of mass-exchange processes and, hence, and on the efficiency of fluorination process, as such, the fluorine supply to the interaction surface, the speed of reaction of fluorination and the speed of removal of hexafluoride from the reaction zone.

\footnotetext{
*Corresponding author: brend989@gmail.com
} 


\section{The mathematical model}

Suppose the limiting stage of the process of fluorination is the fluorine feed to the interaction surface; hence, the concentration of fluorine on the surface becomes equal to zero, and the equation of mass transfer can be written down as:

$$
d M / d \tau=\alpha \cdot \beta_{F} \cdot C_{F} \cdot S,
$$

where $M-$ the mass of the fluorinated tungsten; $\alpha$ - stoichiometric coefficient of reaction (1); $\beta_{F}$ - diffusion coefficient for fluorine; $C_{F}$ - concentration of fluorine in the main gas stream; $S$ - the area of fluorination of solid phase.

Another limiting case is when the limiting stage is the fluorination process as such; then the fluorine feed to the surface would exert practically no impact. Hence, the intensity of the fluorination process of can be written down in the form of speed of the reaction of fluorination:

$$
d M / d \tau=\rho_{w} \cdot V \cdot S
$$

where $\rho_{w}-$ the true density of metal tungsten and $V$ - on the speed of fluorination reaction.

Suppose the stage of diffusive removal of tungsten hexafluoride from the fluorination surface is the limiting factor and the process of fluorination exerts no visible impact on the general speed of process, then the concentration of the key component $\left(W F_{6}\right)$ on the surface of interaction will be almost equal to a unit. At the same time, the speed of the process of fluorination will be defined by the intensity of external mass transfer:

$$
d M / d \tau=\beta_{W F} \cdot\left(1-C_{W F}\right) \cdot S
$$

where $\beta_{W F}$ - diffusion coefficient of the key component of the mix; $C_{W F}$ - concentration of tungsten hexafluoride in the main gas stream.

In case the diffusive and kinetic factors have an identical order of influence on the intensity of the process of fluorination, the concentration of the key component on the surface of interaction is less than the extreme concentration equal to a unit, and the concentration of fluorine on this surface is lower relative to that in the main stream. The concentrations of gas stream components on the surface of fluorination can be determined from the condition of equality between the general speed of fluorination process and the sum of speeds of individual processes:

$$
d M / d \tau=\alpha \cdot \beta_{F} \cdot\left(C_{F}-C_{F}^{\prime}\right) \cdot S=\beta_{W F} \cdot\left(C_{W F}^{\prime}-C_{W F}\right) \cdot S=\rho_{w} \cdot V \cdot S,
$$

where $C^{\prime}{ }_{F}$ and $C^{\prime}{ }_{W F}$ - concentration corresponding a mix component on the fluorination surface.

Thus, the mathematical model based on a system of differential equations in partial derivatives, which are similar to continuity equations and transfer equations derived for components of velocity vector, enthalpy and concentration of the key component of the mix, may simulate accurately the processes of producing gaseous tungsten hexafluoride and, given the boundary conditions, may also have numerical solution. Taking into account the balance ratios (2), it is sufficient to obtain algorithm for calculating the speed of reaction of fluorination of $V$ in order to express the concentration of components in the mix of the fluorination surface, using the characteristics of the stream in the region under consideration, with iterations concluding the computational problem. 


\section{Results and discussion}

The formula for calculating the speed of reaction of fluorination [1] is as follows:

$$
V=K \cdot C_{F}^{\prime},
$$

where $K$ - a constant of fluorination reaction speed.

The latter constant will generally have the same value in certain situations. It is pertinent to cite herein the opinion of other workers: "A difference between the temperature on the reacting surface and the temperature of the gas stream will give the reaction speed of tungsten fluorination: the higher the difference of temperatures, the greater the reaction speed" [2]. The table of constants presented in [3] illustrates the dependence of constants of the reaction of fluorination on temperature in the reaction zone $(T)$ on the one hand and the particle size distribution $(\delta)$ of metal tungsten powder. A regression analysis was made of the available experimental; on the base of results the following functional dependence was obtained for the constant of fluorination reaction speed:

$$
K(\delta, T)=180 \cdot \delta^{-0.073} \cdot e^{-3970 / T}
$$

Using formulas (2), (3) and (4), a closed system of differential equations was obtained which form the basis of the mathematical model for addressing the process of fluorination of metal tungsten. For an assessment the operability of the model can be assessed from the results of test calculations presented in fig. 1 .

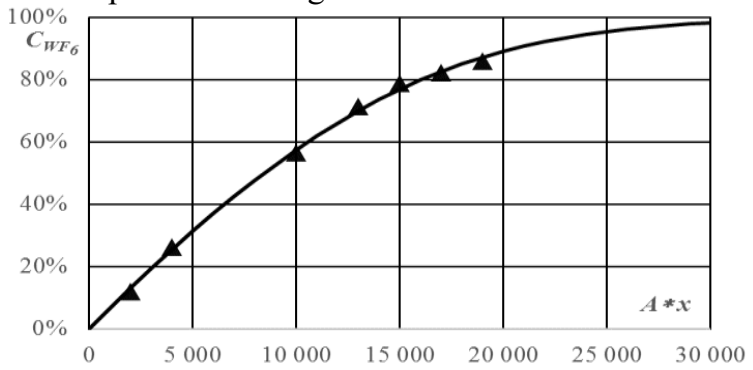

Fig. 1. Concentration of the key component of the mixture from the reaction zone length, continuous line - data calculated in the frame of model; $\boldsymbol{\Delta}$ - data reported in $[4,5]$.

A close match is evidently observed between the calculated and experimental data. This suggests that the proposed model can be applied for choosing optimum conditions of implementation of processes of fluorination of refractory metal powders.

\section{References}

1. Yu. M. Korolev, V. I. Stolyarov, Restoration of fluorides of refractory metals hydrogen (Moscow, Metallurgy, 1981)

2. Patent No. 2310608 - Way of receiving hexafluoride of tungsten. Authors: A. A. Galatian, A. G. Krupin, S. A. Kuzminykh, V. V. Lazarchuk, E. P. Marinenko, A. I. Mines., V. A. Khokhlov (Date of the publication 11/20/2007)

3. Yu. M. Korolev, Int. Symposium on Inorganic fluorides: Chemistry and Technology (TPU Publishing, Tomsk, 2014)

4. T.Sh. Agnokov, Yu.M. Korolev, M.F. Svidersky, and etc., Chemistry and technology of molybdenum and tungsten, Nalchik, KBGU 4 (1978)

5. T.Sh. Agnokov, Yu.M. Korolev, M.F. Svidersky, and etc., Chemistry and technology of molybdenum and tungsten, Nalchik, KBGU 5 (1979) 